\title{
Dachtler-Freiler, Irmgard
}

\section{Neuer Lehrplan - und was nun? Basisüberlegungen zur Lernwirksamkeit des neuen Lehrplans}

Haushalt in Bildung \& Forschung 5 (2016) 4, S. 61-72

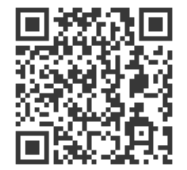

Quellenangabe/ Reference:

Dachtler-Freiler, Irmgard: Neuer Lehrplan - und was nun? Basisüberlegungen zur Lernwirksamkeit des neuen Lehrplans - In: Haushalt in Bildung \& Forschung 5 (2016) 4, S. 61-72 - URN: urn:nbn:de:0111-pedocs-203406 - DOI: 10.25656/01:20340

https://nbn-resolving.org/urn:nbn:de:0111-pedocs-203406

https://doi.org/10.25656/01:20340

in Kooperation mit / in cooperation with:

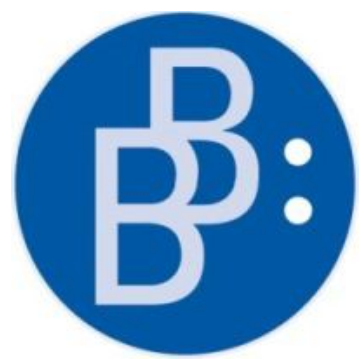

https://www.budrich.de

\section{Nutzungsbedingungen}

Gewährt wird ein nicht exklusives, nicht übertragbares, persönliches und beschränktes Recht auf Nutzung dieses Dokuments. Dieses Dokument ist ausschließlich für den persönlichen, nicht-kommerziellen Gebrauch bestimmt. Die Nutzung stellt keine Übertragung des Eigentumsrechts an diesem Dokument dar und gilt vorbehaltlich der folgenden Einschränkungen: Auf sämtlichen Kopien dieses Dokuments müssen alle Urheberrechtshinweise und sonstigen Hinweise auf gesetzlichen Schutz beibehalten werden. Sie dürfen dieses Dokument nicht in irgendeiner Weise abändern, noch dürfen Sie dieses Dokument für öffentliche oder kommerzielle Zwecke vervielfältigen, öffentlich ausstellen, aufführen, vertreiben oder anderweitig nutzen.

Mit der Verwendung dieses Dokuments erkennen Sie die Nutzungsbedingungen an.

\section{Terms of use}

We grant a non-exclusive, non-transferable, individual and limited right to using this document.

This document is solely intended for your personal, non-commercial use. Use of this document does not include any transfer of property rights and it is conditional to the following limitations: All of the copies of this documents mus retain all copyright information and other information regarding legal protection. You are not allowed to alter this document in any way, to copy it for public or commercial purposes, to exhibit the document in public, to perform, distribute or otherwise use the document in public.

By using this particular document, you accept the above-stated conditions of use.

\section{Kontakt / Contact:}

\section{peDOCS}

DIPF | Leibniz-Institut für Bildungsforschung und Bildungsinformation Informationszentrum (IZ) Bildung

E-Mail:pedocs@dipf.de

Internet: www.pedocs.de

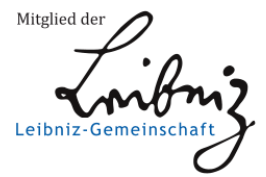




\section{Jahrgang} Heft 4

2016

旁

๓

$\bullet$

号

$\bullet$

돈

용

『্冖

$\frac{\bar{T}}{\Phi}$

$\frac{9}{2}$

정

은

$\stackrel{ \pm}{\frac{1}{2}}$

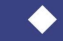

c

$\frac{0}{1}$

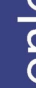

$\xi$

ह

N

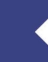

$=$

s

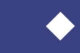

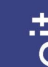

$\frac{2}{2}$

항

ISSN 2193-8806

\section{Haushalt in \\ Forschung}

\section{Lernwirksamkeitsmessung I}

Theoriebildung \& Messinstrumente
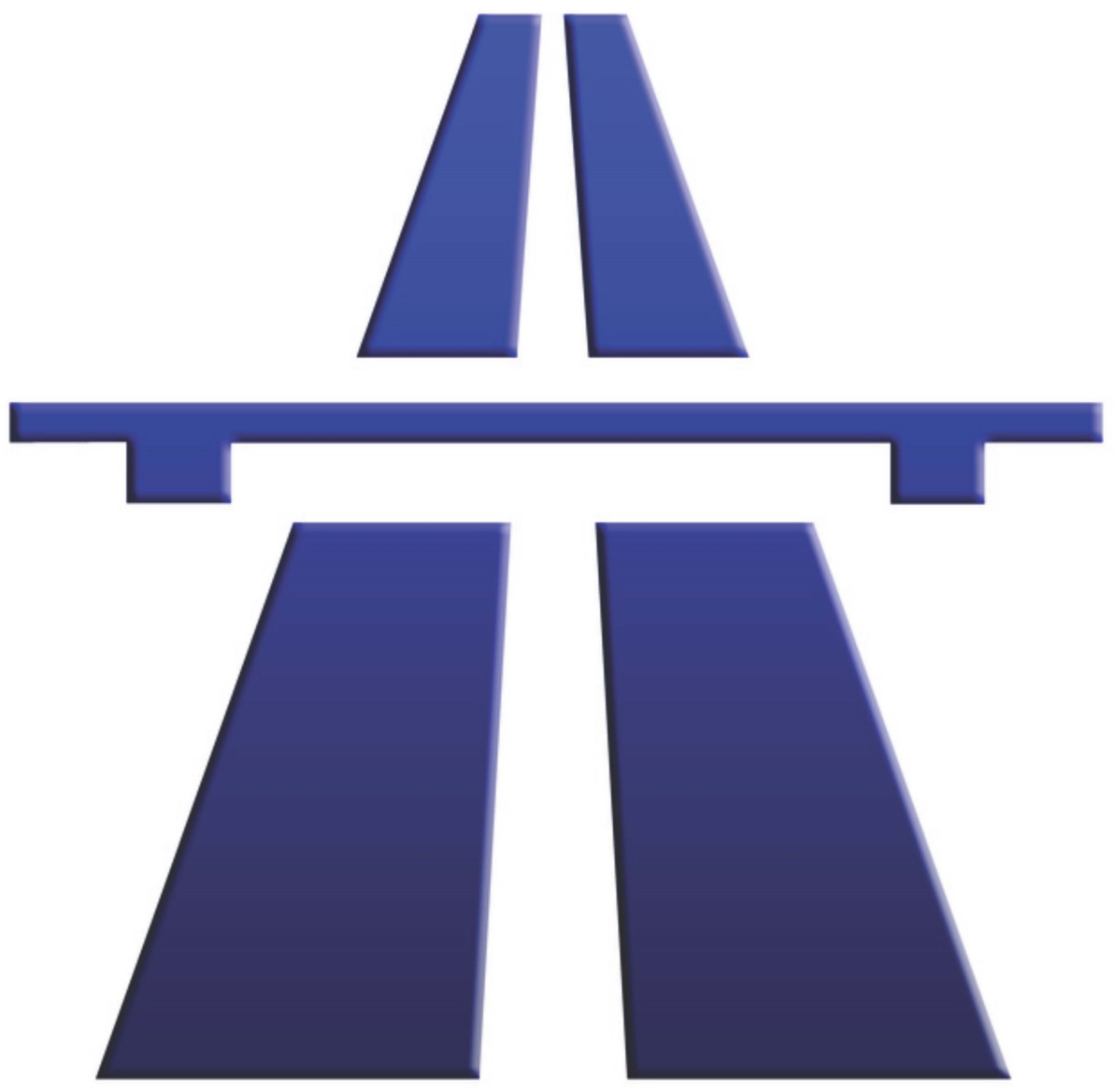


\section{Inhaltsverzeichnis}

Ursula Buchner

Editorial. 2

Werner Brandl

Lernstand erheben, Lernwirksamkeit feststellen,

Lernleistung beurteilen - eine Skizze des Bezugssystems

Gabriele Danninger

Neurodidaktik und Lernwirksamkeit im Lernfeld Ernährung.... 25

Sabine Albert

Die Bedeutung der reflexiven Selbstforschung für die Professionalisierung von Lehrpersonen

Sandro Biaggi \& Claudia Wespi

Professionskompetenzen fördern mit eigenen Videos aus dem Praktikum -

Einblicke in Erfahrungen von Studierenden und Dozierenden

Irmgard Dachtler-Freiler

Neuer Lehrplan - und was nun? Basisüberlegungen zur Lernwirksamkeit des neuen Lehrplans 61

Martina Überall, Maria Lerchbaumer, Christa Meliss \& Birgit Wild

Nachhaltigkeit schmeckt!

Ursula Buchner \& Maria Magdalena Fritz

Lernen im Fach Gesundheit und Soziales: Was wirkt?

Karin Lindner

Langfristige Auswirkungen von Auslandspraktika

auf die berufliche Entwicklung.

Julia Hirsch

Rezension: Essen und Ernährungsbildung in der KiTa 123 
Kompetenzorientierter Lehrplan |

Irmgard Dachtler-Freiler

\section{Neuer Lehrplan - und was nun? Basisüberlegungen zur Lernwirksamkeit des neuen Lehrplans}

Dieses Kapitel stellt die Grundlagen für den Teilbereich Ernährung des Unterrichtsgegenstandes Haushaltsökonomie und Ernährung im neuen Lehrplan der Allgemeinbildenden höheren Schule in Österreich in der 10. Schulstufe vor und will klären, wie dieser lernwirksam umgesetzt werden kann.

Schlüsselwörter: Lehrplan AHS - Haushaltsökonomie und Ernährung, Ernährungsbildung, handlungsorientierter Unterricht, Lernwirksamkeit

\section{Grundlagen für den neuen Lehrplan}

Der neue semestrierte, kompetenzorientierte Lehrplan der Allgemeinbildenden höheren Schulen (AHS) wurde im Bundesgesetzblatt II Nr. 219/2016 am 9. August 2016 verlautbart.

Die Neugestaltung der Lehrpläne ist auf Grund der Oberstufenreform notwendig geworden. Im Zuge dessen wurde der Unterrichtsgegenstand Haushaltsökonomie und Ernährung so gestaltet, dass die Lehrinhalte der Haushaltsökonomie nunmehr nur in der 9. Schulstufe unterrichtet werden und die Lehrinhalte der Ernährung ausschließlich in der 10. Schulstufe. Mit der Neugestaltung der Lehrpläne werden verschiedene Maßnahmen gesetzt, die u.a. zu einer Verdichtung der Lernaktivität bei den Lernenden führen und die Eigenverantwortung der Schülerinnen und Schüler stärken sollen. In der neuen Oberstufe (NOST) ist eine Semestrierung der Lehrpläne ab der 10. Schulstufe vorgesehen. Das bedeutet, dass die Bildungs- und Lehraufgaben inklusive des Lehrstoffs einzelnen Semestern zuzuordnen und in diesen zu unterrichten sind. Dies soll eine lernorganisatorische Verbesserung und eine kontinuierliche Leistungserbringung der Lernenden bewirken. Nach jedem Winter- und Sommersemester wird nunmehr ein Zeugnis ausgestellt (bisher war im Wintersemester eine Schulnachricht vorgesehen). Sollte das Zeugnis in einem Unterrichtsgegenstand ein Nicht genügend bzw. ein Nicht beurteilt ausweisen, so sind auf einem Beiblatt zum Semesterzeugnis die nicht erreichten Bildungs- und Lehraufgaben von den Lehrenden einzutragen, wobei Teilleistungen (im Sinn einzelner, erreichter Kompetenzen) anerkannt werden (BM:BF, 2015). Durch diese Neuerungen mussten die Lehrpläne umgestaltet werden, wobei nicht vorgesehen war, die lernzielorientierten Formulierungen des Lehrplans 2004 gänzlich zu verwerfen. Daher spiegeln sich die 


\section{Kompetenzorientierter Lehrplan}

Lernziele des Lehrplans 2004 im Fachbereich Ernährung großteils in den Kompetenzen des Lehrplans 2016 wider.

Als Grundlage für alle Gegenstände des neuen Lehrplans musste ein Kompetenzmodell entwickelt werden. Dem Kompetenzmodell liegt der Referenzrahmen für Ernährungs- und Verbraucherbildung in Österreich zu Grunde (Thematisches Netzwerk Ernährung, 2015). Dieser ist in Anlehnung an die Entwicklung des europäischen Kerncurriculums entstanden, in dem jene fachspezifischen Kompetenzen definiert sind, die eine verbindliche Grundausbildung in den Fachbereichen Haushaltsökonomie und Ernährung sicherstellen sollen. Dieses Kompetenzmodell findet sich auch in der Handreichung für die mündliche kompetenzorientierte Reifeprüfung im Gegenstand Haushaltsökonomie und Ernährung wieder. Das Kompetenzmodell für Haushaltsökonomie und Ernährung sieht fünf übergeordnete semesterübergreifende Kompetenzbereiche vor.

\section{Übergeordnete semesterübergreifende Kompetenzbereiche:}

- Bewusstsein über das eigene Konsumverhalten entwickeln, konsumspezifische Informationen beschaffen und nach Qualitätskriterien bewerten

- Ressourcen verantwortungsbewusst managen

- Das Konzept des Consumer Citizenship erkennen, reflektieren und in Entscheidungen einbinden

- Das eigene Essverhalten erkennen, reflektieren und bewerten sowie Zusammenhänge zwischen Ernährungsgewohnheiten, Kulturen, Gesundheit und Wohlbefinden verstehen

- Sich vollwertig und nachhaltig ernähren. (BGB1. II Nr. 219/2016)

In den einzelnen Semestern werden diese Kompetenzbereiche durch Teilkompetenzen mittels Operatoren beschrieben, so wird eine Verknüpfung von Handlung und Lehrstoff hergestellt. Ein Auszug aus dem Lehrplan soll dies verdeutlichen:

\section{Klasse: 3. Semester - Modul 1}

Das eigene Essverhalten erkennen, reflektieren und bewerten sowie Zusammenhänge zwischen Ernährungsgewohnheiten, Kulturen, Gesundheit und Wohlbefinden verstehen:

- Essgewohnheiten in Bezug auf Tradition, Kultur und Religion nennen

- Zusammenhänge zwischen Ernährungsgewohnheiten und Gesundheit beschreiben sowie verantwortliches Handeln bezüglich sozialem, psychischem und körperlichem Wohlbefinden diskutieren

- den eigenen Energie- und Nährstoffbedarf berechnen

- soziokulturelle und sensorische Einflüsse auf die eigenen Ernährungsgewohnheiten wahrnehmen und beschreiben. (BGB1. II Nr. 219/2016) 


\section{Kompetenzorientierter Lehrplan |}

In der Präambel zum Unterrichtsfach Haushaltsökonomie und Ernährung im Lehrplan der AHS werden die groben Zielsetzungen des Gegenstandes erläutert sowie Beiträge dieses Faches zu anderen Bildungsbereichen (z. B. Mensch und Gesellschaft, Natur und Technik u. a.) näher ausgeführt. Im Anschluss sollen die didaktischen Grundsätze Lehrende dazu anregen einen kompetenz- und handlungsorientierten Unterricht zu entwickeln.

\section{Widerspruch? Wissen - Bildung - Kompetenz}

Man lernt doch nur für die Schule und nicht fürs Leben, weil man ja nicht wissen kann, was das Leben in den nächsten Jahrzehnten bringen wird, meinte der römische Philosoph Seneca vor langer Zeit.

Der Ende Juni 2016 präsentierte Nationale Bildungsbericht Österreich 2015 befasst sich u.a. mit der Lernwirksamkeit des Schulsystems und versteht darunter längerfristige Effekte der Leistungen des schulischen Unterrichts für die Absolventinnen und Absolventen selbst als auch für die Gesellschaft als Ganzes. Es geht also um die tatsächlich verfügbaren Fähigkeiten im Alltagshandeln, um eine erfolgreiche Teilhabe an allen Bereichen des gesellschaftlichen Lebens, sei es in der Arbeitswelt oder in privaten und öffentlichen Handlungsfeldern, zu garantieren (BM:BF \& Bundesinstitut bifie, S. 2007).

Was muss man wirklich wissen? Und - was ist eigentlich Wissen bzw. Bildung?

Wissen ist mehr als der Lehrstoff eines Unterrichtsgegenstandsbereichs oder die im Internet abrufbaren Informationen einzelner Daten. Wissen setzt sich aus mehreren Elementen zusammen: kennen-verstehen-bewerten. Wissen befähigt erläutern zu können, was und warum etwas so oder anders ist. Um den Sinn und die Zusammenhänge zu verstehen, bedarf es der Fähigkeit selbst zu denken. Selbst zu denken erfordert aber Neugier sowie Fähigkeiten wie das Sammeln und Abwägen von Argumenten, das kritische Überprüfen von Hypothesen und letztlich lebenspraktische Klugheit, die man sich nur durch lebenslanges Lernen aneignen kann. Lebenspraktische Klugheit erwirbt man sich durch sinnvolle Auseinandersetzung mit diesem Wissen und Integration dieses Wissens in das eigene Persönlichkeitskonzept, damit verantwortungsvolles Handeln möglich wird. Peter Drucker spricht vom Wissensarbeiter als „gebildete Person“, die das Wissen in der Gegenwart anzuwenden weiß und für die Gestaltung der Zukunft nutzen kann (Liessmann, 2006, S. 29-34).

Gebildet sein heißt, sich selbst und die Welt angemessen zu verstehen (Adorno, 1980, S. 112).

Das bedeutet, sich selbst ganzheitlich zu formen, seine Begabungen und Talente zu einer Individualität zu entfalten, um an der Gesellschaft partizipieren zu können. 


\section{Kompetenzorientierter Lehrplan}

Bildung ist „das Programm der Menschwerdung durch die geistige Arbeit an sich und an der Welt" (Liessmann, 2006, S. 59).

Selbsterkenntnis und Freiheit des Individuums sind somit die Endziele der Bildung (ebda S. 56). Die Schule hat daher die wichtige Aufgabe, das kritische Denken und die Selbstreflexion zu fördern und so die Lernenden zur Urteilsfähigkeit zu führen. Aber wie kann man als Pädagogin oder Pädagoge darauf hinarbeiten, dass Lernende diese Fähigkeit erwerben und wie kann man das messen?

Folgt man Philosophen wie Precht (2013), Liessmann (2006) lässt sich Bildung weder messen noch normieren. Die schulische Bildung steht für Liessmann unter dem Diktat der Standardisierung und Kompetenzorientierung.

Aber hier kommt man bei der schulischen Arbeit ins Dilemma. Wie sollen Lehrende den Spagat zwischen Kompetenzorientierung und „Bildungsarbeit“" schaffen? Wenn man Kompetenz als Fähigkeit einer Person begreift, die in bestimmten Situationen sinnvoll $\mathrm{zu}$ handeln imstande ist und damit verschiedene Aufgaben und Lebenssituationen bewältigt (Roth, 1971), dann kommt man der Sache, um die es in der Bildungspolitik in den letzten Jahren geht - um die Ausrichtung von Lehr- und Lernprozessen auf den Erwerb von Kompetenzen und somit zur erhofften Qualitätssteigerung des Unterrichts - schon näher. Roth nennt drei Bereiche, die einen mündigen und gebildeten Menschen ausmachen: Sach-, Selbst- und Sozialkompetenz.

\section{Welches Wissen soll kompetenzorientierter Unterricht fördern?}

Der Leitbegriff Kompetenzorientierung ist eine Weiterentwicklung der Lernzielorientierung. Dabei geht es nicht mehr darum ,was durchgenommen wurde“, sondern das konkrete Wissen und Können der Schülerinnen und Schüler steht nunmehr im Vordergrund. Kompetenz wird als handelnder Umgang mit Wissen gesehen und ist daher ein anzustrebendes Bildungsziel.

Anderson und Krathwohl (2001) beschreiben unterschiedliche Wissensaspekte, die im Zusammenspiel erst Kompetenz ergeben:

- Deklaratives Wissen umfasst Fakten, Prinzipien, Theorien, Modelle, Strukturen.

- Prozedurales Wissen ist praktisch nutzbares Wissen und wird in Anwendungsprozessen sichtbar.

- Strategisches, metakognitives Wissen ist das Wissen über das eigene Denken und Handeln, das Wissensmanagement.

Für Weinert (2001) kommen dazu noch Motivation und Volition. Durch den Bezug der verschiedenen Wissensaspekte zueinander und aufeinander zeigt sich, dass Kompetenzentwicklung mehr ist als Wissensvermittlung und Qualifizierung. Nach Heyse, Erpenbeck und Max (2004, S. 8) charakterisieren Kompetenzen „die Fähigkeiten von 


\section{Kompetenzorientierter Lehrplan |}

Menschen, sich in offenen und unüberschaubaren, komplexen und dynamischen Situationen selbstorganisiert zurechtzufinden“.

\section{Beitrag zur Ernährungsbildung}

\section{Was will der facheinschlägige Unterricht des Gegenstandes Ernährung be- wirken?}

Folgt man dem bereits Erwähnten, dann muss es Ziel des Unterrichts sein, Schülerinnen und Schüler zu kritischem Denken und zur Reflexionsfähigkeit anzuregen, Möglichkeiten für problemorientiertes Lernen zu bieten und die Bereitschaft zu verantwortungsvollem Handeln zu unterstützen. Kompetenzen zur eigenen Gesunderhaltung und zum nachhaltigen Konsum können nicht allein auf Wissensvermittlung beruhen, hier spielt auch die motivational-volitionale Dimension (Weinert 2001) eine wichtige Rolle.

Ernährungsbildung muss daher den gesamten Menschen in den Fokus nehmen und versuchen, bei Schülerinnen und Schülern jene Fach-, Methoden-, Sozial- und Personalkompetenz aufzubauen, damit diese imstande sind, sich gesund zu ernähren und darüber hinaus im gesamtgesellschaftlichen und welternährungswirtschaftlichen Kontext richtige Entscheidungen zu treffen und so das Problem Ernährung selbstverantwortlich in den einzelnen Lebensbereichen und Lebensphasen zufriedenstellend lösen zu können (Thematisches Netzwerk, 2009, S. 10). Gesundheitserziehung alleine kann daher für die Autorin keine Legitimation für den Fachunterricht Ernährung darstellen.

\section{Wie kommen Lernergebnisse bei Lernenden zustande?}

„Unter Lernen versteht man den absichtlichen und den beiläufigen, individuellen oder kollektiven Erwerb von geistigen, körperlichen, sozialen Kenntnissen, Fähigkeiten und Fertigkeiten" (https://de.wikipedia.org/wiki/Lernen). Lernen ist ein aktiver, ganzheitlicher, individueller und konstruktiver Prozess, der auf Weiterentwicklung abzielt, wobei Selbstregulierungsprozesse die Aufgabe haben, Kognition, Motivation und Verhalten zu steuern (Leopold \& Leutner, 2004, S. 365). Lernen ist also die Voraussetzung, dass Bildung erworben werden kann, wenn Bildung bedeutet, sich selbst zu formen und seine Begabungen und Talente zu entfalten.

Der kompetenzorientierte Lehrplan soll handlungsorientiertes, problemlösendes, kreativitätsförderndes Lernen und einen schülerzentrierten Unterricht in einer anregenden Lernumgebung ermöglichen.

Aufgaben der Verfasserinnen und Verfasser des neuen, kompetenzorientierten Lehrplans war es, die Lernziele zu operationalisieren. Die Bildungs- und Lehraufgaben sind in der Handlungsdimension abgebildet und werden mittels Operatoren formuliert, die erläutern, welche Kompetenzen die Schülerinnen und Schüler erreichen sollen. Der Lehrstoff findet sich in der Inhaltsdimension. 


\section{Kompetenzorientierter Lehrplan}

Durch die Operationalisierung werden Fähigkeitsbereiche sichtbar gemacht. Somit soll Transparenz über den Grad der Erreichung der Lernziele bzw. „,der Kompetenz" erzielt werden.

Wie lassen sich Lehr- und Lernziele von Lehrenden in Handlungsziele bzw. Kompetenzen für Lernende umwandeln?

Le(h)rnziele sind organisatorisches Prinzip für die Zielorientierung des Unterrichtens, Handlungsziele werden didaktisch entwickelt. Le(h)rn- und Handlungsziele müssen aufeinander abgestimmt werden, indem Handlungsprodukte bzw. Handlungssituationen zur Schnittmenge von Le(h)rn- und Handlungszielen werden (Gudjons, 2001, S. 145). Dabei darf die funktionale Verknüpfung von Denken und Handeln nicht außer Acht gelassen werden.

Denken beinhaltet Einspeicherungs-, Erinnerungs-, Reaktions-, Urteils- und Entscheidungsprozesse. Handeln besteht aus drei Komponenten: planen-realisierenkontrollieren und bewerten (Gudjons, 2001, S. 48). Durch das Handeln werden Informationen, Kenntnisse und Einsichten in eine kognitive Struktur gebracht. Aus dem Aufbau kognitiver Strukturen im Zusammenhang mit dem Handeln von Lernenden ist begründbar, dass der Aufbau von Denk- und Handlungsstrukturen einem unzusammenhängenden, sog. ,trägem Wissen“ vorzuziehen ist.

Nur eine Handlungsvorstellung kann das praktische Handeln nicht ersetzen, weil durch die effektive Handlung (Operation) leichter gelernt und verstanden wird als bei reinen Gedankenexperimenten. Dies kann man u.a. am Beispiel der Käseerzeugung nachvollziehen, wo Schülerinnen und Schüler selbsttätig Käse herstellen und so die Stadien der Erzeugung anschaulich erleben.

Am Aufbau praktischer Handlungsschemata wird aufgezeigt, was dann beim Denken auf der abstrakten Ebene abläuft. Abstrakte Operationen können als abstrakte Handlungen gesehen werden. Denken und Handeln bzw. Theorie und Praxis gehören daher zusammen, wobei die sprachliche Unterstützung hierbei von Relevanz ist (Gudjons, 2001, S. 52-59).

Handlungsorientierter Unterricht muss so vorbereitet werden, dass Kompetenzen in Schritten aufgebaut werden können bis hin zur mehrperspektivischen Handlungskompetenz und Selbstverantwortung der Schülerinnen und Schüler.

Ohne dass auf die weit verbreitete Literatur verschiedener Autoren, die sich umfassend mit unterschiedlichen Theorien zum handlungsorientierten Unterricht befassten, näher eingegangen werden kann, soll hier ein kurzer Diskurs zum handlungsorientierten Unterricht unternommen werden.

Der handlungsorientierte Unterricht beruht auf der Grundannahme, dass der Mensch ein lern- und vernunftfähiges Wesen ist, das sich kritisch mit sich und der Welt auseinandersetzen will (Gudjons, 2001, S. 40), was, wie bereits oben erläutert, der Anspruch an Bildung ist. 


\section{Kompetenzorientierter Lehrplan |}

\section{Wie lässt sich handlungsorientierter Unterricht theoretisch begründen?}

Die materialistische Aneignungstheorie (von Wygotski begründet und von Leontjew sowie Galperin weiterentwickelt) geht von einer Wechselwirkung von Gegenständen, Erscheinungen und Prozessen in der Welt und den Wahrnehmungen, Empfindungen und Begriffen des menschlichen Bewusstseins aus. Es findet eine Umwandlung von materiellen Handlungen in geistige Strukturen statt. Im Gebrauch von Werkzeugen, der Sprache und in der Ausbildung geistiger Handlungen reproduziert sich die Gesellschaft. Aneignung kann als menschliches Lernen bezeichnet werden. Der Mensch verändert sich und die Umwelt bei diesem Prozess (Gudjons, 2001, S. 43-46).

Die Handlungsregulationstheorie (von Hacker und Volpert entwickelt) will die Entfremdung der Schule vom ,wirklichen Leben“ überwinden, in dem konkrete Handlungsprozesse im Unterricht ausgeführt werden. Handlungen sind zielgerichtet und werden bewusst durchgeführt. Sie haben eine motivationale Grundlage. Handlungen sind bedürfnis- und situationsbedingte psychische oder physische Aktivitäten. Handlungen haben eine Struktur - plan-do-check - und damit eine Antizipations-, Realisations-, und Reflexions- und Bewertungskomponente. Mit diesen Komponenten wird die Regulationsstruktur begründet. Damit soll ein kognitiver Strukturaufbau mit immer differenzierter werdendem Handlungsrepertoire einhergehen und Denken und Handeln verschränkt werden (Dulisch, 1986, S. 64-81).

Die kognitive Handlungstheorie fußt auf Aebli. Eine effektive Handlung muss verinnerlicht, systematisiert und sprachlich kodiert werden, damit sie auf andere Problemstellungen übertragen werden kann. Die Sprache spielt als Stütze eine wichtige Rolle, sodass Handlungen später ohne konkretes Tun wiedergegeben werden können. Kognitive Strukturen und Handeln sind somit eng verknüpft, da sich Denken aus dem Handeln entwickelt und wieder auf dieses steuernd zurückwirkt (Aebli, 1981).

Die Lern- und Motivationspsychologie ist zwar keine Theorie, aber auch hier steht das Lernen durch Handeln im Fokus. Laut einer Untersuchung der American Audiovisuell Society über menschliche Behaltensleistungen behalten wir $20 \%$ Gehörtes, 30\% Gesehenes, 80\% selbst Formuliertes und 90\% selbst Getanes. Wenn viele Sinne (auditiver, visueller, taktiler) durch Ausprobieren, Untersuchen, Herstellen usw. angesprochen werden, wächst die Motivation zu lernen. Hier kann man einen Zusammenhang zwischen Kognition und Emotion herstellen, weil das limbische System im menschlichen Großhirn eng mit dem Denken, dem Lernen und der Verarbeitung von Emotionen verknüpft ist (Gudjons, 2001, S. 59-67).

Was spricht für einen handlungsorientierten, fächerübergreifenden Unterricht, der die Entwicklung von Kompetenzen fördern soll?

Wenn schulisches Lernen ein zielorientierter, versprachlichter und reflektierter Handlungsprozess ist, dann steht nicht der Lehrstoff per se im Mittelpunkt des Ge- 


\section{Kompetenzorientierter Lehrplan}

schehens, sondern die Aneignung desselben über die auf Planung, Realisierung und Erkenntnis ausgerichtete Problemlösung, die zur Lebensbewältigung der Schülerinnen und Schüler in der Zukunft führen soll. Die Schule soll zur Lebensbewältigung befähigen (s. Bildung) und Sachwissen in zielgerichtetes Handlungswissen transformieren.

Die Intention des kompetenzorientierten Lehrplans ist ein Abwenden vom lehrerzentrierten Frontalunterricht des „Beibringens und Vermittelns“. Handlungsorientiert und schülerzentriert unterrichten heißt Bezug nehmen auf die Vorerfahrungen der Schülerinnen und Schüler, damit im Zusammenhang mit konkreten Handlungserfahrungen das Lernen (d. h. Zugewinn von Erkenntnissen, Dispositionen, Kompetenzen und Einsichten in Dinge) angemessen stattfindet.

\section{Lernwirksamkeit}

Lernen, das bei Schülerinnen und Schülern zu nachhaltigen Kompetenzen führen soll, bedarf eines konstruktiven Lernverständnisses bei Lehrenden sowie eines entsprechend vorbereiteten und durchgeführten Unterrichts. Lehrpersonen müssen einerseits professionelles Fachwissen und andererseits didaktische Fähigkeiten aufweisen, um lernwirksame Lerngelegenheiten im Unterricht bieten zu können sowie diagnostische Fähigkeiten, damit adressatenbezogen bei den Lernenden Kognitionsund Konzeptveränderungen möglich werden.

Wenn Lernende sich aktiv denkend und elaborierend mit Sachverhalten auseinandersetzen sollen, sind dazu anregende Lernumgebungen und Unterrichtssituationen herzustellen, die einer Zielformulierung auf einer komplexen Stufe nach Anderson und Krathwohl entsprechen, z. B. analysieren-entwickeln-bewerten.

Das Lösen von komplexen Problemstellungen, das von einem ,gebildeten Menschen" verlangt wird, braucht situative, authentische Lerngelegenheiten, wo eine individuelle Ko-Konstruktion des Wissens im sozialen Austausch möglich wird. Die Konfrontation mit anderen Sichtweisen, Meinungen und Ideen ist von besonderer Bedeutung für die Erweiterung des Wissens.

\section{Worauf beruft sich dieses Verständnis von Lernen?}

Diesen didaktischen Grundsätzen liegen erkenntnis- und handlungsleitend das aktive, forschende und handlungsorientierte Lernen sowie der „cognitive apprenticeshipAnsatz ${ }^{\text {“1 }}$ zu Grunde (Collins, Brown \& Newman, 1989).

Die Lernwirksamkeit bzw. der Lernertrag hängt aber nicht nur von didaktischen Kunstgriffen und der Lehrerpersönlichkeit ab, sondern hier spielen auch andere Einflussfaktoren hinein. $\mathrm{Zu}$ den personenbezogenen Voraussetzungen zählen kognitive Vorgaben, das Lernpotenzial sowie das Erwartungs-Wertmodell und die Selbstwirksamkeitsannahme des Lernenden, welche für die Motivation von Bedeutung sind, aber auch das familiäre Umfeld (Lipowsky, 2014, S. 513-515). 
Kompetenzorientierter Lehrplan |

\section{Ein vorläufiges Schlusswort ...}

Die Wirksamkeit von Lehrplänen wird in der Forschung seit langem skeptisch beurteilt, aber die Bildungspolitik erwartet sich mit der Einführung des neuen, kompetenzorientierten Lehrplans für die Allgemeinbildende höhere Schule in Österreich eine Orientierung am „Learning-Outcome“, eine entsprechende Unterrichtserteilung vom intendierten Lernziel her und somit eine Steigerung der Unterrichtsqualität.

Der Output wird hierbei als Kompetenz definiert, welche sich die Lernenden aneignen sollen. Kompetenzen sind Persönlichkeitseigenschaften, die Leistungen ermöglichen. Kompetenzen sind nicht direkt sichtbar, sondern drücken sich nur in Ergebnissen von Handlungen aus, in der Performanz. Die erzielte Leistung hängt daher nicht nur von den Fähigkeiten einer Person ab, sondern auch von ihrer Anstrengungsbereitschaft (Motivation und Volition). Performanz bezieht sich auf eine Leistung in einer konkreten Situation und ist beobachtbar, daher messbar und bewertbar.

Für das Messen von Leistungen sind Leistungsindikatoren bzw. Messkriterien operational zu konstruieren. Diese nehmen den Charakter von Handlungskriterien im Lehrplan an und werden mit aktiven Verben, z. B. erklären-vergleichen-bewerten, beschrieben. Hierbei ist eine hierarchische Komplexität (Anderson \& Krathwohl 2001 nach Bloom) gegeben. Diese Handlungskriterien werden zu Normen, wobei fälschlicher Weise das Gemessene oft als Bildung verstanden wird (Koch, 2010, S. 29).

\section{Repräsentiert das Gemessene das Gemeinte?}

Hier sind wir wieder beim Thema: Lassen sich Kompetenzen messen? Lässt sich Bildung messen?

Wie schon erwähnt, lassen sich Kompetenzen nur über längerfristige, erfolgreiche Lernprozesse erwerben. Sie setzen sich aus zusammenhängenden Komponenten wie Wissen, die Fähigkeit komplexe Aufgaben zu bewältigen sowie aus Fertigkeiten zusammen und enthalten Aspekte von Erfahrung, Motivation und persönlichen Einstellungen. Das Reproduzieren einer Formel, z. B. $\mathrm{C}_{6} \mathrm{H}_{12} \mathrm{O}_{6}$ für Glucose, ist leicht überprüfbar. Aber die Überprüfung einer Kompetenz in ihrer Ausprägung kann nur mit Hilfe geeigneter Problemstellungen festgestellt werden.

Shahram Azizi Ghanbari ersetzt den ,kompetenzorientierten Unterricht“ durch einen ,zielvaliden Unterricht“. Er stellt fest, dass mit „Kompetenz“ in der Regel das Lernergebnis der Lernenden gemeint ist. Die Lehrerinnen und Lehrer haben demnach so zu unterrichten, dass die Schülerinnen und Schüler genau das lernen, was sie beim Prüfen bzw. Messen auch können sollen. Es sind daher solche Aufgaben zu stellen, die zielvalide den „Kompetenzgrad“ festlegen (Ghanbari, 2014, S. 35-42). Die Verfasserinnen des Lehrplans für den Fachbereich Ernährung haben die Indikatoren zum Messen der Leistung diesbezüglich sorgfältig ausgewählt. 


\section{Kompetenzorientierter Lehrplan}

Handlungsorientierter Unterricht soll dabei helfen, die zu vermittelnden Lernziele bzw. Kompetenzen, also den gewünschten Output zu erreichen. Handlungsorientierter Unterricht muss sich daher seines Bezugs zum Lehrplan bewusst sein, ein strukturiertes Konzept aufweisen und darf keinesfalls in ungeplantes „learing by doing“ ausarten.

Handlungsorientierter Unterricht muss garantieren, dass die am Einzelfall erworbenen Kenntnisse, Fertigkeiten und Einsichten auch auf neue Sachverhalte und Situationen transferiert werden können, damit er nachhaltig wirkt. Wenn Lernende in der realen Lebenswelt handlungsfähig werden sollen, müssen demnach mehrperspektivische Handlungsentwürfe aufgebaut werden. Erst durch den Erwerb von umfassender Handlungsfähigkeit für sich selbst und im Hinblick auf die Gesellschaft in der Domäne Ernährung kann man von nachhaltiger Ernährungsbildung sprechen.

\section{Anmerkung}

1 Cognitive apprenticeship ist eine Methode, die kognitive Prozesse für die Lernenden sichtbar machen soll. Diese Methode hat einen sozial-konstruktivistisch begründeten Ansatz. Ziel dieser Methode ist, das eigene Handeln kritisch zu hinterfragen und die eigene Problemlösungskompetenz auf andere Situationen übertragen zu können.

\section{Literatur}

Adorno, T. W. (1980). Theorie der Halbbildung. Gesammelte Schriften Bd. 8/1. Frankfurt/Main: Suhrkamp Verlag.

Aebli, H. (1981). Grundformen des Lernens. Eine allgemeine Didaktik auf kognitionspsychologischer Grundlage. Stuttgart: Klett-Cotta.

Anderson, L. W. \& Krathwohl, D. R. (2001). A taxonomy for learning and assessing: a revision of Bloom's taxonomy of educational objectives. New York: Abridged Edition.

BGB1. II Nr. 219/2016 vom 9.8.2016.

BM:BF (Hrsg.). (2015). Die neue Oberstufe. Individuell und kompetenzorientiert. Die rechtlichen Grundlagen. Verfügbar unter www.bmbf.gv.at [01.08.2016].

BM:BF \& Bundesinstitut bifie (Hrsg.). (2015). Nationaler Bildungsbericht Österreich 2015, Band 1 .

Collins, A., Brown, J. S., \& Newman, S. (1989). Cognitive apprenticeship: teaching the crafts of reading, writing and mathematics. In L. B. Resnick (Ed.), Knowing learning and instruction: Essay in honor of Robert Glaser. Hillsdale. New York: Erlbaum.

Dulisch, F. (1986). Lernen als Form menschlichen Handelns. Eine handlungstheoretisch orientierte Analyse von Lernprozessen unter besonderer Berücksichtigung 


\section{Kompetenzorientierter Lehrplan |}

des Selbststeuerungsaspektes. Wirtschafts- und Berufspädagogische Schriften, Bd. 2. Bergisch Gladbach: Hobein.

Ghanbari, S. A. (2014). Die Kompetenz, kompetenzorientiert zu unterrichten. Doppeldecker Kompetenzförderung 3, S. 35-42.

Gudjons, H. (2001). Handlungsorientiert lehren und lernen. Bad Heilbrunn: Klinkhardt.

Heyse, V., Erpenbeck, J. \& Max, H. (2004). Kompetenzen erkennen, bilanzieren und entwickeln. Münster: Waxmann.

Koch, L. (2010). Messbarkeit als Norm.

http://www.diezeitschrift.de/42010/koch1001.pdf

Leopold, C. \& Leutner, D. (2004). Selbstregulierendes Lernen und seine Förderung durch prozessorientiertes Training. In J. Doll \& M. Prenzel (Hrsg.), Bildungsqualität von Schule: Lehrerprofessionalisierung, Unterrichtsentwicklung und Schülerförderung als Strategien der Qualitätsverbesserung. Münster: Waxmann.

Liessmann, K. P. (2006). Theorie der Unbildung. Wien: Paul Zsolnay Verlag.

Lipowsky, F. (2014). Theoretische Perspektiven und empirische Befunde zur Wirksamkeit von Lehrerfort- und -weiterbildung. In E. Terhart, H. Bennewitz \& M. Rothland (Hrsg.), Handbuch der Forschung zum Lehrerberuf. Münster: Waxmann.

Precht, R.D. (2013): Anna, die Schule und der liebe Gott: Der Verrat des Bildungssystems an unseren Kindern. München: Goldmann.

Roth, H. (1971). Pädagogische Anthropologie. Hannover: Schroedel.

Thematisches Netzwerk Ernährung (Hrsg.). (2009). Ernährungsbildung in Österreich. Stand und Entwicklung der Ausbildungssituation.

http://www.thematischesnetzwerkernaehrung.at/downloads/Ernaehrungsbildung \%20in\%20Oesterreich_Stand\%202009.pdf

Thematisches Netzwerk Ernährung (Hrsg.). (2015). Referenzrahmen für die Ernährungs- und Verbraucherbildung in Österreich.

http://www.theamatischesnetzwerkernaehrung.at

Weinert, F. E. (2001). Vergleichende Leistungsmessung in Schulen - Eine umstrittene Selbstverständlichkeit. In F. E. Weinert (Hrsg.). Leistungsmessungen in Schulen. Weinheim: Beltz.

Wikipedia. Lernen. https://de.wikipedia.org/wiki/Lernen 


\section{Kompetenzorientierter Lehrplan}

\section{Verfasserin}

$\operatorname{RgR}^{\text {in }}$. Mag ${ }^{\text {a. }}$ Irmgard Dachtler-Freiler

Pädagogische Hochschule Wien

Institut für Berufsbildung

Grenzackerstraße 18

A-1100 Wien

E-Mail: irmgard.dachtlerfreiler@gmail.com

Internet: www.phwien.ac.at 